\title{
Factors affecting uptake of antenatal HIV testing in London: results of a multicentre study
}

Diana M Gibb, Sandra E MacDonagh, Ramyani Gupta, Pat A Tookey, Catherine S Peckham, A E Ades

\begin{abstract}
Objectives: To measure the uptake of antenatal HIV testing and determine its relation to risk of HIV and to screening practices.

Design: Multicentre prospective questionnaire study. Subjects: Pregnant women attending six maternity units.

Setting: Inner London, 1995-6.

Main outcome measures: Uptake of testing by risk factors for HIV, ethnicity, and factors about the antenatal interview.

Results: All units had a "universal offer" policy for HIV testing. In five units forms were completed for 18791 (88\%) of 21247 pregnant women. The sixth unit, where the response rate was too low to assess uptake, was excluded from the analysis. Uptake ranged from $3.4 \%$ to $51.2 \%$ (overall $22.9 \%$ ), in parallel with detection of previously undiagnosed infection in pregnant women (4.9-60\%). Controlling for unit, uptake was higher among the 7\% who disclosed risk factors. Among those at low risk, uptake varied by ethnic group (South Asian women 9\%; Latin

American and Mediterranean women 33\%). The relation between uptake and HIV risk category varied greatly across units. Despite increased HIV

seroprevalence in black African women, uptake was similar in this group to that among women at low risk (24\%). Uptake increased 2.1-fold if HIV transmission was discussed. Midwives reported spending 7 (2-15) minutes discussing HIV issues.
\end{abstract}

Conclusions: Uptake of HIV testing was unacceptably low in all units, with maternity unit the strongest predictor. New approaches to antenatal HIV testing are urgently required and uptake should be audited routinely.

\section{Introduction}

Antiretroviral therapy ${ }^{1}$ and avoidance of breastfeeding ${ }^{2}$ can reduce the risk of mother to child transmission of HIV from 25-30\% to 5-8\%. ${ }^{4}$ In the United Kingdom, women who know their HIV status are increasingly taking up these interventions. ${ }^{5}$ Despite national guidelines recommending that antenatal HIV testing should be offered to all women in areas of higher prevalence such as London, ${ }^{6}$ in 1995 only 13 (39\%) of the 33 London maternity units had a policy of offering testing to all pregnant women. ${ }^{7}$ Nationally, detection of previously undiagnosed HIV infection in pregnancy has remained below $10 \%{ }^{8}$ This study reports the offer and uptake of antenatal HIV testing in six London maternity units and examines how these are related to maternal risk of exposure to HIV and screening practices.

\section{Methods}

Six inner London maternity units with high HIV seroprevalence participated in the study. All had a "universal offer" policy, but implementation varied between units (see table 1). Within each unit, data on demographic and obstetric factors, including risk factors for HIV infection, were collected by questionnaire over a 12 month period during 1995 and 1996. Data on the number of women booking for antenatal care from each unit were collected and checked with laboratory data on number of samples sent for rubella antibody testing.

Data were analysed with univariate and multivariate logistic regression models controlling for hospital, risk category, ethnic group, place of booking interview, age, whether HIV was discussed and testing offered, and the interaction between hospital and risk category. Risk category included data on risks disclosed during the antenatal interview, and the categories "black African" and "partner black African," derived from self report of ethnic group and country of birth. The category "exposed in Africa" included any women born in Africa and those who gave their ethnic group as black African.

\section{Results}

One unit was excluded from the analysis (unit F) because only $17 \%$ of forms were returned, making calculation of uptake impossible. In the remaining five units, 21247 women were booked for antenatal care over the period and 18791 forms were returned (response rate of $88 \%$, range $81-100 \%$ ) (table 1 ). Uptake of testing ranged from $3.4 \%$ to $51.2 \%$ (overall mean $22.9 \%$ ). With the exception of unit $\mathrm{C}$, where uptake increased from $22.9 \%$ to $33.6 \%$, no significant trends were observed over the 12 month study period. The detection of previously undiagnosed HIV infection during pregnancy (based on unlinked anonymous surveys) ranged from $4.9 \%$ to $60 \%$ (23\% overall) and increased in parallel with uptake (table 1).

Overall, maternity unit had the greatest influence on uptake of testing $\left(\chi^{2}=2907, \mathrm{df}=4, \mathrm{P}<0.0001\right)$. Length of gestation, the woman's fluency in English, whether she was seen alone or not, or booked in hospital or the community had little or no effect (data not shown). Among women with whom discussion about HIV transmission had taken place, uptake was higher by a factor of 2.1 (95\% confidence interval 1.9 to 2.4) after other factors were controlled for, but in $23 \%$ of booking interviews no such discussion was reported.

Uptake of HIV testing was significantly higher among the $7 \%$ of women who disclosed a risk factor during the antenatal interview. Women who believed their partner was at risk ("partner other risk") or that
See editorial by Mercey

Department of Epidemiology and Public Health, Institute of Child Health, London WCIN 1EH

Diana M Gibb, senior lecturer in epidemiology

Sandra E

MacDonagh, research midwife

Ramyani Gupta, research fellow

Pat A Tookey, research fellow

Catherine $\mathrm{S}$

Peckham,

professor in epidemiology and public health

A E Ades,

reader in epidemiology and public health

Correspondence to: Dr Gibb d.gibb@ich.ucl. ac.uk

BMJ 1998;316:259-61 
Table 1 Characteristics of maternity unit and uptake of HIV testing. Values are numbers (percentages) unless otherwise indicated

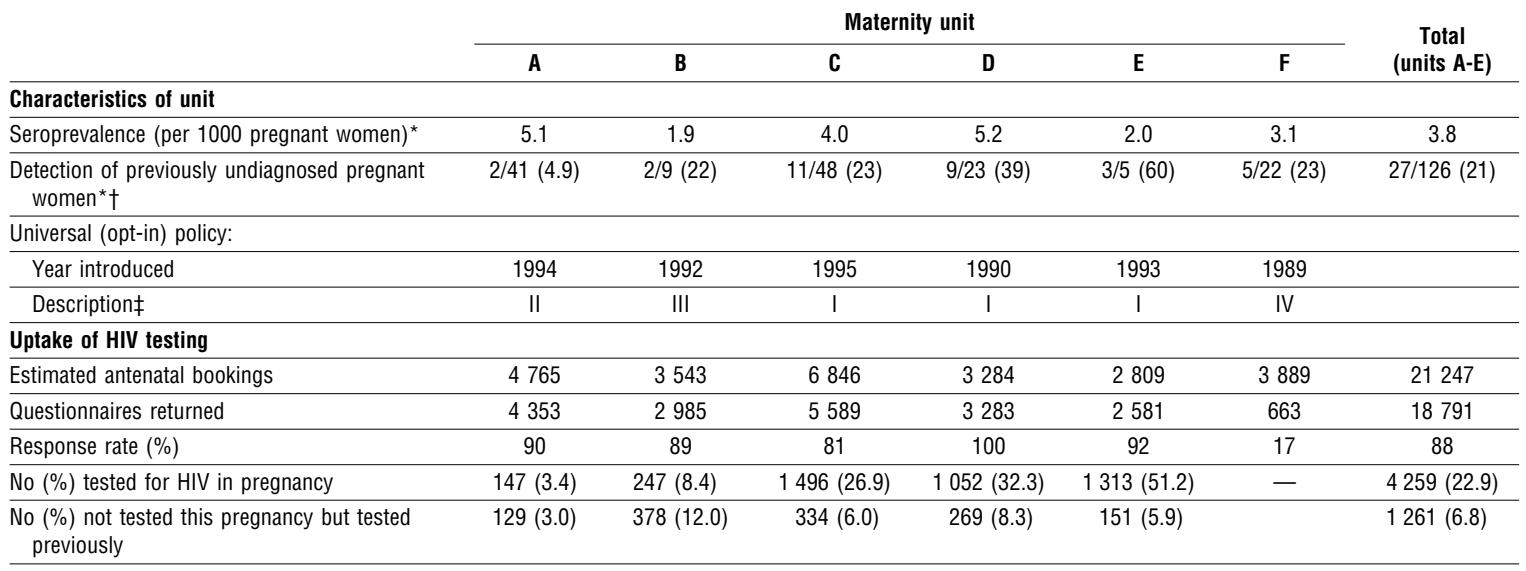

*From unlinked anonymous antenatal survey results and Royal College of Gynaecologists' reports of infected women, 1995 and 1996, Institute for Child Health and Public Health Laboratory Service.

tDetection rate=women identified this pregnancy/(total infected women-women identified before pregnancy).

$\ddagger \mathrm{l}=$ Discussion, test offer, and authorisation by midwife; results at next appointment if negative, recall by post if positive; II=discussion with midwife, referral to HIV counsellor for all women wishing to be tested; test authorisation by counsellor; III=discussion, test offer, and authorisation by midwife; all results by special appointment; IV=discussion with midwife, test offer, and authorisation by obstetrician or counsellor at 16-18 weeks.

they were at risk ("other risk") had the highest uptakes, often citing occupational exposure or multiple partners (table 2). Those disclosing intravenous drug use also had higher uptake. Those who had been exposed in Africa and those who had a partner who was black African had the lowest uptakes. Among those not disclosing risk there were strong ethnic differences, uptake being lowest $(9 \%)$ in women of South Asian ethnic origin and highest (33\%) in women of Mediterranean or Latin American origin.

The relation between risk category and uptake of testing varied considerably among units after other factors were controlled for $\left(\chi^{2}=223, \mathrm{df}=36\right.$, $\mathrm{P}<0.0001)$. The greatest difference in uptake between risk categories was observed in units with the lowest uptake of testing. For example, the greatest difference (56-fold relative uptake) was observed between "partner intravenous drug user" and "no disclosed risk: other" in unit A, which had the lowest overall uptake (3.4\%); conversely, the smallest difference (1.4-fold) was observed between "partner other risk" and "no disclosed risk: other" in unit E, which had the highest overall uptake.
The average time spent by midwives discussing HIV issues was 7 minutes (95\% confidence interval 2 to 15 minutes). This was similar across units and among women accepting testing and those not accepting (7.4 $v 6.8$ minutes). No women in units $\mathrm{D}$ and $\mathrm{E}, 0.6 \%$ of women in unit $\mathrm{B}, 0.8 \%$ in unit $\mathrm{C}$, and all women wanting a test (3.4\%) in unit A saw a counsellor. The mean time taken by counsellors was 20 (3 to 45 ) minutes and was independent of whether a woman was tested.

\section{Discussion}

Uptake of testing by maternity unit

In five major London maternity units with high rates of HIV infection, less than a quarter of women were tested. This compares with 73-99\% uptake reported in France, Scandinavia, and the United States. ${ }^{3}{ }^{10}$ The large differences between units cannot be explained by time spent by midwives discussing HIV issues, which was similar in all units, but may be due to other differences in implementing a "universal offer" policy. Unit A, with the lowest uptake, was the only unit where all women wanting an HIV test had to see a counsellor

Table 2 Percentage uptake of testing and relative uptake in five maternity units (ranked by aggregate unit uptake) by risk category

\begin{tabular}{|c|c|c|c|c|c|c|c|}
\hline \multirow[b]{2}{*}{ Risk category* } & \multirow{2}{*}{$\begin{array}{l}\text { No }(\%) \text { of } \\
\text { women }\end{array}$} & \multicolumn{6}{|c|}{ Percentage uptake (relative uptake $\dagger$ ) by maternity unit } \\
\hline & & A & B & C & D & $\mathrm{E}$ & Total \\
\hline Partner other risk & $328(1.8)$ & $56(52.3)$ & $41(5.9)$ & $67(2.9)$ & $49(1.7)$ & $73(1.4)$ & $57(2.5)$ \\
\hline Woman other risk & $254(1.4)$ & $34(32.2)$ & $32(4.6)$ & $43(1.9)$ & $77(2.6)$ & $69(1.3)$ & $48(2.1)$ \\
\hline Woman intravenous drug user & $98(0.5)$ & $0(0.0)$ & $24(3.5)$ & $24(1.0)$ & $64(2.2)$ & $60(1.1)$ & $43(1.9)$ \\
\hline Partner intravenous drug user & $30(0.2)$ & $60(56.1)$ & $38(5.4)$ & $30(1.3)$ & 0 & $25(0.5)$ & $33(1.5)$ \\
\hline $\begin{array}{l}\text { No disclosed risk: Latin American or } \\
\text { Mediterranean }\end{array}$ & $970(5.2)$ & $4(3.7)$ & $11(1.5)$ & $36(1.6)$ & $40(1.3)$ & $53(1.0)$ & $33(1.5)$ \\
\hline Woman exposed in Africa & $2453(13.2)$ & $6(5.5)$ & $8(1.1)$ & $31(1.4)$ & $37(1.3)$ & $49(0.9)$ & $24(1.1)$ \\
\hline Partner black African & $826(4.4)$ & $3(2.6)$ & $14(2.0)$ & $29(1.2)$ & $34(1.2)$ & $49(0.9)$ & $24(1.1)$ \\
\hline Unknown risk & $366(2.0)$ & 0 & $13(1.9)$ & $39(1.7)$ & $43(1.5)$ & $59(1.1)$ & $28(1.2)$ \\
\hline No disclosed risk: South Asian & $2202(11.8)$ & $1(0.8)$ & $6(0.9)$ & $20(0.9)$ & $25(0.9)$ & $30(0.6)$ & $9(0.4)$ \\
\hline No disclosed risk: otherf & $11086(59.5)$ & $1(1.0)$ & $7(1.0)$ & $23(1.0)$ & $29(1.0)$ & $54(1.0)$ & $22(1.0)$ \\
\hline
\end{tabular}

${ }^{*}$ Composed from data on risks disclosed during antenatal interview and self reported ethnic group and country of birth. If a woman belonged to more than one risk category, she was assigned to the first category in the following hierarchy: woman intravenous drug user; exposed in Africa; woman other risk; partner intravenous drug user; partner black African; partner other risk; unknown risk; no disclosed risk.

tRelative to uptake in risk category "No disclosed risk: other."

$\neq 0 f$ this group, $74 \%$ were white. 
first. The largest differences in uptake by risk category also occurred in this unit but were not due to higher uptake among women known to be at higher risk (exposed in Africa or intravenous drug user), as might be expected with a selective policy. Instead, differences were due to higher uptake among those women (only $3 \%$ of the antenatal population) who regarded themselves or their partners as at risk. Unit A was in practice implementing an "on request" policy. In contrast, the highest overall uptake, in unit E, was associated with little variation across risk categories; this was the unit with the most "universal offer" policy.

\section{Uptake among women exposed in Africa}

Of particular concern, because of their much higher seroprevalence, ${ }^{11}$ was the finding that uptake among women exposed in Africa or with partners exposed in Africa was no higher than among most women with no disclosed risk. It is unclear whether these women were unaware of their increased risk or, if aware, were unwilling to be tested or assumed they were being tested when they were not. ${ }^{12}$ Midwives may also be reluctant to offer HIV testing to African women because of concerns about perceived discrimination.

\section{Detection of previously undiagnosed HIV infection}

Detection of previously undiagnosed infection was higher in units with higher uptake, but overall nearly $80 \%$ of previously undiagnosed infected women went through pregnancy without being tested, unaware of their status and unable to benefit from interventions to reduce transmission of HIV from mother to child. A major reason for universal rather than selective HIV testing in areas of higher prevalence is to ensure high uptake among women at highest risk. Unless a concerted effort is made to increase overall uptake to much higher levels than those in this study, it could be argued that it might be better to direct resources to improving uptake in black African women despite the problems of perceived discrimination. An analysis of economic factors to determine how decisions on screening strategies should be tailored to patterns of HIV seroprevalence and distribution of risk categories in different parts of the United Kingdom is being undertaken. This will inform policy and assist in defining appropriate new strategies in London and in areas of low or intermediate seroprevalence outside London. Routine audit of HIV testing uptake, which in 1995 was lacking in all units, ${ }^{7}$ is a prerequisite for the routine evaluation of urgently required new approaches to antenatal HIV testing.

We thank the midwives, counsellors, obstetricians, and microbiologists from the participating units and particularly members of the Uptake Study Steering Committee for their input and support throughout the study. This study was funded by the Department of Health, as is the unlinked anonymous seroprevalence monitoring programme. We thank Danielle Mercey, Susan Bewley, and Angus Nicoll for comments on drafts of the paper.

Members of the Uptake Steering Group are Susan Bewley, consultant obstetrician; Sandra Dick, midwife/HIV counsellor; Jane Kennedy, midwife/counsellor; Danielle Mercey, consultant genitourinary physician; Richard Smith, consultant obstetrician; C Hudson, emeritus professor of obstetrics and gynaecology; Glyn Evans, consultant obstetrician; Ruby Edwards, head midwife; and Angus Nicoll, consultant epidemiologist.

Contributors: DMG wrote the original grant application, set up the initial logistics for the study, supervised SEMacD,
Key messages

- In five major maternity units in inner London, all with "universal offer" policies, fewer than 1 in 4 women were tested for HIV during pregnancy in 1995-6

- Maternity unit was the most important factor determining uptake of HIV testing, which ranged from $3 \%$ to $51 \%$, in parallel with detection of previously undiagnosed infection during pregnancy

- Maternity units with the lowest uptake of antenatal HIV testing had the largest differences in uptake between groups of women with different risk factors for HIV

- Uptake of testing among women exposed in Africa was no higher than among women with no disclosed risk, despite much higher HIV seroprevalence in the former group

- New approaches to antenatal HIV testing are urgently required; ongoing routine audit of the uptake of antenatal HIV testing is essential if higher uptake rates are to be achieved and maintained

contributed to data analysis, took the lead in writing the paper, and is the guarantor. SEMacD carried out the study, supervised data entry, and was involved in data analysis and writing the paper. RG analysed the study and contributed to writing the results. PAT supported SEMacD and commented on the paper. CSP contributed to the grant application and commented on the paper. AEA made a major contribution to the grant application, had most ideas about data analysis, supervised RG, and contributed to writing the paper.

Funding: PAT is supported by AVERT (the AIDS Education and Research Trust) and DMG by the Medical Research Council. The study was funded by the Department of Health; the views expressed in this paper are those of the authors alone.

Conflict of interest: None.

1 Connor EM, Mofenson LM. Zidovudine for the reduction of perinatal human immunodeficiency virus transmission: Pediatric AIDS Clinical Trials Group Protocol 076-results and treatment recommendations. Pediatr Infect Dis J1995; 14:536-41.

2 Dunn DT, Newell ML, Ades AE, Peckham CS. Risk of human immunodeficiency virus type 1 transmission through breastfeeding. Lancet 1992;340:585-58.

3 Fiscus SA, Adimora AA, Schoenbach VJ, Lim W, McKinnery R, Rupar D, et al. Perinatal HIV infection and the effect of zidovudine therapy on transmission in rural and urban counties. JAMA 1996;275:1483-8.

4 Mayaux MJ, Teglas JP, Mandelbrot L, Berrebi A, Gallais H, Matheron S. Acceptability and impact of zidovudine prevention on mother-to-child HIV-1 transmission in France. J Pediatr 1998 (in press).

5 Gibb DM, MacDonagh SE, Tookey PA, Duong T, Nicoll A, Goldberg DJ, et al. Uptake of interventions to reduce mother-to-child transmission of HIV in United Kingdom and Ireland. AIDS 1997;11:F53-8.

6 Department of Health. Guidelines for offering voluntary named HIV antibody testing to women receiving antenatal care. London: $\mathrm{DoH}, 1994$.

7 MacDonagh SE, Masters J, Helps BA, Tookey PA, Ades AE, Gibb DM. Descriptive survey of antenatal HIV testing in London: policy, uptake, and detection. BMJ 1996;313:532-3.

8 Nicoll A, McGarrigle C, Brady T, Ades AE, Tookey PA, Duong T. Detection of HIV-1 among pregnant women in the United Kingdom: results from public health surveillance 1988-95. BMJ 1998;316:253-8.

9 Rey D, Carrieri M, Obadia Y, Pradier C, Moatti JP. Interest and limits of mandatory HIV prenatal screening: the south-eastern experience, 19921994. Br J Obstet Gynaecol 1998 (in press).

10 Lindgren S, Bohlin AB, Forsgren M, Arneborn M, Ottenblad C, Lidman $\mathrm{K}$, et al. Screening for HIV-1 antibodies in pregnancy: results from the Swedish national programme. BMJ 1993;307:1447-51.

11 De Cock KM, Low N. HIV and AIDS, other sexually transmitted diseases, and tuberculosis in ethnic minorities in United Kingdom: is surveillance serving its purpose? BMJ 1997;314:1747-51.

12 Kahtan S. Antenatal HIV testing. Anonymous testing misleads patients. BMJ 1993;306:1479.

(Accepted 7 November 1997) 